\title{
Chronic E-Cigarette Use Increases Neutrophil Elastase and Matrix Metalloprotease Levels in the Lung
}

\author{
Arunava Ghosh ${ }^{1}$, Raymond D. Coakley ${ }^{1}$, Andrew J. Ghio ${ }^{2}$, Marianne S. Muhlebach ${ }^{1}$, Charles R. Esther, Jr. ${ }^{1}$, \\ Neil E. Alexis ${ }^{3}$, and Robert Tarran ${ }^{1,4}$ \\ ${ }^{1}$ Marsico Lung Institute, ${ }^{2}$ Environmental Protection Agency, ${ }^{3}$ Center for Environmental Medicine, Asthma, and Lung Biology, \\ and ${ }^{4}$ Department of Cell Biology and Physiology, The University of North Carolina at Chapel Hill, Chapel Hill, North Carolina
}

\begin{abstract}
Rationale: Proteolysis is a key aspect of the lung's innate immune system. Proteases, including neutrophil elastase and MMPs (matrix metalloproteases), modulate cell signaling, inflammation, tissue remodeling, and leukocyte recruitment via cleavage of their target proteins. Excessive proteolysis occurs with chronic tobacco use and is causative for bronchiectasis and emphysema. The effect of e-cigarettes (vaping) on proteolysis is unknown.

Objectives: We used protease levels as biomarkers of harm to determine the impact of vaping on the lung.

Methods: We performed research bronchoscopies on healthy nonsmokers, cigarette smokers, and e-cigarette users (vapers), and determined protease levels in BAL. In parallel, we studied the effects of e-cigarette components on protease secretion in isolated human blood neutrophils and BAL-derived macrophages. We also analyzed the nicotine concentration in induced sputum and BAL.
\end{abstract}

Measurements and Main Results: Neutrophil elastase, MMP-2, and MMP-9 activities and protein levels were equally elevated in both vapers' and smokers' BAL relative to nonsmokers. In contrast, antiprotease levels were unchanged. We also found that exposure of isolated neutrophils and macrophages to nicotine elicited dose-dependent increases in protease release. After vaping, measurable levels of nicotine were detectable in sputum and BAL, which corresponded to the half-maximal effective concentration values for protease release seen in immune cells.

Conclusions: We conclude that vaping induces nicotine-dependent protease release from resident pulmonary immune cells. Thus, chronic vaping disrupts the protease-antiprotease balance by increasing proteolysis in lung, which may place vapers at risk of developing chronic lung disease. These data indicate that vaping may not be safer than tobacco smoking.

Keywords: vaping; protease; BAL; sputum; nicotine
Tobacco smoking is responsible for chronic respiratory disease, cardiovascular disease, and several types of cancer (1). Electronic nicotine delivery systems (i.e., e-cigarettes) are noncombustible tobacco alternatives that aerosolize nicotine and flavoring agents in a propylene glycol and vegetable glycerin (PG/VG) vehicle. They were originally envisaged as a tobacco cessation tool (2), but whether or not they help people to quit tobacco use is unclear (3-5). Vaping e-cigarettes has been perceived by the general public and some professional bodies as a safer alternative to tobacco smoking (6), and a recent report from Public Health England reiterated previous advice that e-cigarettes are $95 \%$ safer than smoking and that people should switch from tobacco to e-cigarettes (7). However, this is controversial because there are conflicting data regarding their safety and, to date, no robust long-term exposure data exist $(8,9)$. In contrast, a recent European Respiratory Society task force concluded that, because the long-term effects of vaping are unknown, there is no evidence that they are safer than tobacco and that, based on current knowledge, negative health effects cannot be ruled out (10). Given that some professional bodies are now recommending e-cigarettes as a safe alternative to tobacco and are asking for them to be prescribed clinically (11), it is

(Received in original form March 13, 2019; accepted in final form August 7, 2019)

Author Contributions: A.G., A.J.G., M.S.M., and C.R.E. performed experiments. R.D.C. interviewed patients and performed bronchoscopies. A.G., C.R.E., and R.T. analyzed data. A.G., N.E.A., and R.T. designed experiments. A.G. and R.T. wrote the manuscript, and all other authors edited or approved the manuscript.

Correspondence and requests for reprints should be addressed to Robert Tarran, Ph.D., Marsico Hall, 125 Mason Farm Road, University of North Carolina at Chapel Hill, Chapel Hill, NC 27599. E-mail: robert_tarran@med.unc.edu.

This article has an online supplement, which is accessible from this issue's table of contents at www.atsjournals.org.

Am J Respir Crit Care Med Vol 200, Iss 11, pp 1392-1401, Dec 1, 2019

Copyright @ 2019 by the American Thoracic Society

Originally Published in Press as DOI: 10.1164/rccm.201903-0615OC on August 7, 2019

Internet address: www.atsjournals.org 


\section{At a Glance Commentary}

\section{Scientific Knowledge on the}

Subject: The effects of combustible tobacco products on lung protease-antiprotease balance are well known. However, the effects of chronic e-cigarette use (vaping) on the protease-antiprotease balance in the lung are poorly understood.

\section{What This Study Adds to the Field:}

We demonstrate that vapers' lungs have elevated levels of neutrophil elastase, MMP-2, and MMP-9 with no change in antiprotease levels. In vitro exposure of neutrophil and macrophages to nicotine elicited protease release. Vaping resulted in sputum and BAL nicotine concentrations sufficient to cause protease release from macrophages.

imperative to understand whether or not they are actually safer than conventional tobacco products.

Proteolysis is a form of post-translational modification that can degrade unwanted proteins and/or modify protein function $(12,13)$. As such, there are multiple proteases present both intracellularly and extracellularly whose activity is tightly regulated by a series of antiproteases for overall positive benefit. In the lung, changes in proteolysis do not necessarily result in detrimental outcomes. For example, these changes can alter antimicrobial activity, airway hydration and mucus clearance, inflammation, and cell signaling, and can facilitate tissue turnover as part of normal homeostasis. However, during multiple types of chronic lung disease, protease-antiprotease imbalance occurs, and the subsequent increase in proteolysis contributes to pathogenesis (14-17). The association between combustible tobacco products and increased proteolysis has been well established (18-20), and previous studies revealed enhanced protease levels and activity in the lungs of cigarette smokers (21-24). For example, increased MMP (matrix metalloprotease) expression and basement membrane degradation has been shown in smokers and patients with chronic obstructive pulmonary disease (COPD) (25). Similarly, increased proteolysis drives bronchiectasis in cystic fibrosis, lung disease, and in alpha-1 antitrypsin deficiency $(26,27)$. Abnormal proteolysis also plays a role in tumor progression and metastasis by facilitating tissue remodeling (28).

Recently, it has been demonstrated that chronic e-cigarette use alters the bronchial proteome (29). E-cigarette use has also been linked with immunosuppression in the upper airways and enhanced levels of detoxifying proteins and mucins in sputum $(30,31)$. Inhaled e-liquid aerosols are thought to be deposited in the bronchoalveolar region (32). Therefore, we postulated that secreted proteases could serve as well-validated biomarkers of harm because they are causal for the development of chronic lung disease when in imbalance with antiproteases.

However, limited information is available on the actual effects of chronic e-cigarette use on the protease-antiprotease balance of vapers' lungs. Thus, we tested the hypothesis that chronic e-cigarette exposure leads to similar increases in secreted lung proteases as seen with chronic tobacco exposure. To test this hypothesis, we performed research bronchoscopies on "never-smokers," current tobacco smokers, and a cohort of e-cigarette users that included both never-smokers and former tobacco smokers. We measured BAL protease levels. In parallel, we also studied the effects of e-liquid components on protease secretion from relevant immune cells in vitro.

\section{Methods}

\section{Study Design}

We performed research bronchoscopy on nonsmokers, smokers, and vapers as previously published (29). All subjects provided informed consent and completed a smoking diary to document tobacco product use in the last 2 weeks prior to sample acquisition. Details of sample acquisition are provided in the online supplement.

\section{Protease Evaluation in BAL Samples}

The relative expression level of different proteases was evaluated using Western blotting of concentrated BAL in agematched subjects. For measurement of protease activity in BAL samples, we used fluorescent peptides. MMP-2/9 activity was also evaluated using zymography. Details are provided in the online supplement.

\section{Statistical Analysis}

All the data sets were checked for normal distribution. Parametric or nonparametric analysis was chosen based on the distribution of the data; one-way ANOVA was used to compare the groups, and $P \leqslant$ 0.05 was considered significant. The specific post hoc tests used are reported in figure legends. GraphPad Prism software was used to measure the half-maximal effective concentration $\left(\mathrm{EC}_{50}\right)$ values by plotting $\log$ concentration of nicotine with the measured outcome.

Additional methods are listed in the online supplement.

\section{Results}

\section{Demographics of Nonsmokers, Smokers, and Vapers}

We performed research bronchoscopies on healthy subjects, including never-smokers, cigarette smokers, and e-cigarette users (vapers) as described (29). All subjects completed a smoking diary and questionnaire to determine their current and former smoking and vaping habits. Subject demographics and smokin and vaping habits are shown in Tables 1 and E1 in the online supplement, respectively. No significant difference in subject demographics or lung function was observed between groups. All nonsmokers were never-smokers, and all tobacco smokers had significant and regular cigarette use. Nine out of 14 vapers had a history of former tobacco use and had switched to e-cigarettes for at least 6 months prior to bronchoscopy, and the remainder were never-smokers. To confirm tobacco and vape use, we measured serum levels of nicotine, cotinine, and hydroxycotinine. For nonsmokers, these compounds were at or below the limit of detection (Table E2). As previously described (29), serum from smokers and vapers had significantly increased levels of nicotine and its metabolites compared with nonsmokers, and no significant differences in metabolite levels were observed between smokers and vapers (see Table E2). We also performed cytological analysis of BAL. Immune cell numbers were not different across the groups, indicating that we were studying smokers and vapers who did not have significant pathology (see Table 1).

\section{BAL from Smokers and Vapers Exhibit Increased Neutrophil Elastase and MMP Levels but Unchanged Protease Inhibitor Levels}

We performed Western blot analysis to determine the relative levels of $\mathrm{NE}$ 
Table 1. Subject Demographics and Cytology Analysis of BAL Samples

\begin{tabular}{lccc}
\hline & Nonsmokers & Smokers & Vapers \\
& & & \\
Sex, F/M & $10 / 4$ & $6 / 8$ & $4 / 10$ \\
Ethnicity, African & $5 / 1 / 7 / 1$ & $7 / 0 / 7 / 0$ & $1 / 3 / 10 / 0$ \\
$\quad$ American/Asian/white/other & & & \\
Age at bronchoscopy, yr & $25.79 \pm 7.29$ & $29.50 \pm 5.59$ & $26.07 \pm 8.30$ \\
Height, cm & $165.46 \pm 7.97$ & $171.86 \pm 11.42$ & $172.70 \pm 8.35$ \\
Weight, kg & $72.47 \pm 21.51$ & $82.04 \pm 18.63$ & $88.73 \pm 20.35$ \\
Body mass index, kg/m ${ }^{2}$ & $26.17 \pm 5.87$ & $27.84 \pm 6.05$ & $29.79 \pm 6.60$ \\
FVC, \% predicted & $100.65 \pm 22.47$ & $110.50 \pm 8.72$ & $100.64 \pm 8.69$ \\
FEV 1 \% predicted & $102.86 \pm 13.51$ & $106.36 \pm 9.43$ & $100.07 \pm 6.96$ \\
Polymorphonuclear cells, \% & $2.88 \pm 2.61$ & $1.99 \pm 1.69$ & $3.13 \pm 2.58$ \\
Eosinophils, \% & $0.47 \pm 0.94$ & $0.26 \pm 0.43$ & $0.32 \pm 0.48$ \\
Lymphocytes, \% & $0.07 \pm 0.17$ & $0.00 \pm 0.00$ & $0.00 \pm 0.00$ \\
Bronchial epithelial cells, \% & $0.47 \pm 0.65$ & $0.19 \pm 0.33$ & $0.46 \pm 0.65$ \\
Squamous epithelial cells, \% & $0.17 \pm 0.25$ & $0.30 \pm 0.42$ & $0.17 \pm 0.23$ \\
Macrophages, \% & $96.11 \pm 2.40$ & $97.56 \pm 1.78$ & $96.10 \pm 2.89$ \\
\hline
\end{tabular}

Data were provided as mean \pm SD except where otherwise indicated.

(neutrophil elastase), MMP-2, and MMP-9 in BAL. These data were normalized to albumin as loading controls. NE, MMP-2, and MMP-9 protein levels were significantly elevated in both smokers and vapers compared with nonsmokers (Figures 1A-1D). These increases were sex independent, and there was no significant difference in protease levels between vapers and smokers. We next evaluated BAL antiprotease levels. Accordingly, we probed for antiproteases; namely, alpha-1 antitrypsin and secretory leukocyte protease inhibitor, which can both inhibit $\mathrm{NE}$; and tissue inhibitors of MMP-1 and MMP-2, which can inhibit MMPs. Despite seeing significant increases in NE and MMP levels in smokers' and vapers' BAL, we did not detect any significant changes in protease inhibitor levels (see Figures 1E-1I). Because many vapers have previously used tobacco products, one cannot be sure whether any observed changes were due to current vaping or prior tobacco use. However, 5 out of 14 vapers reported that they had never used tobacco products before becoming e-cigarette users (see Table E1). We therefore assessed their levels of NE, MMP-2, and MMP-9 protein levels relative to nonsmokers. Despite their lack of prior cigarette use, all three proteases were significantly elevated relative to nonsmokers, indicating that the increase in BAL protease levels was caused by vaping rather than first-hand exposure to tobacco (Figure E2).

Protease levels were elevated, whereas antiprotease levels were unchanged, suggesting that net protease activity had increased. Accordingly, we then tested for changes in BAL protease activity using two orthogonal approaches. First, we measured gelatinolytic activity using zymography. Because MMP-2 and MMP-9 proteases are collagenases that can degrade basement membranes, we measured their ability to cleave a gelatin gel. Indeed, both smokers and vapers showed significantly increased activity relative to nonsmokers (Figure 2). Again, these increases were sex-independent and not different between smokers and vapers. Second, we compared MMP-2 and MMP9 activity in BAL from vapers who never smoked versus nonsmokers and again found that they were significantly increased (see Figure E2).

Next, we measured the ability of endogenous BAL proteases to cleave peptide-based fluorogenic substrates that were specific to 12 different proteases (Table E3 and Figure E3) in a subset of BALs that were sex-matched and age-matched (Table E4). All protease activities were normalized to the total BAL protein content. The peptide dinitrophenyl-Pro-Leu-Gly-MetTrp-Ser-Arg is cleaved by both MMP-2 and MMP-9, and cleavage of this peptide was significantly upregulated in both vapers and smokers (see Figure E3A). NE cleaves SucAla-Ala-Ala-MCA and, similarly, cleavage was significantly upregulated in both vapers and smokers (see Figure E3B). In contrast, cathepsin B activity was elevated only in smokers compared with nonsmokers (see Figure E3C). No differences were observed in the activities of the other proteases tested (see Figures E3D-E3I), and again no sex-dependent differences were observed (see Figures E3A-E3I).

\section{Nicotine Induces Protease Secretion in Human Neutrophils and Macrophages In Vitro}

Neutrophils and macrophages are two major inflammatory cell types involved in lung defense (33). We did not observe significantly altered cell counts or neutrophilia in either vapers or smokers BAL (see Table 1), suggesting that the increased protease levels (see Figures 1 and 2) were not simply due to altered immune cell numbers. Therefore, to understand how vaping leads to elevated protease levels in vapers' BAL, we exposed isolated immune cells to e-cigarette components. Because neutrophil numbers were low in our BAL samples, we isolated peripheral blood neutrophils by negative magnetic selection and immediately exposed them to the base e-liquid components; that is, PG/VG in a $55: 45$ volume/volume ratio $\pm 18 \mathrm{mg} / \mathrm{ml}$ nicotine or an equivalent amount of nicotine in media. We exposed neutrophils to e-liquid components for 4 hours, as indicated, and subsequently collected the media to measure NE and GAPDH levels by Western blot. Elevated levels of NE and GAPDH were detected in the media of neutrophils incubated with $3 \%$ PG/VG and nicotine, or with nicotine alone (Figures $3 \mathrm{~A}$ and $3 \mathrm{~B}$ ). Further confirmation of NE release was obtained via the fluorogenic substrate assay, and we observed significantly increased NE activity after exposure to $3.3 \mathrm{mM}$ nicotine (see Figure 3C). This response was also independent of PG/VG. The presence of GAPDH in the media after treatment with nicotine (see Figures $3 \mathrm{~A}$ and $3 \mathrm{~B}$ ) were indicative of possible neutrophil lysis. Three percent PG/VG has a concentration of $408 \mathrm{mM}$, which when added to media caused an increase in osmolarity from approximately $300 \mathrm{mOsm}$ (media alone) to greater than $700 \mathrm{mOsm}$. Because hyperosmotic solutions can cause osmotic stress and/or cell lysis, we also treated neutrophils to media with $408 \mathrm{mM}$ mannitol as an osmotic control (Table E5). However, despite the increase in osmolarity, the mannitol control did not affect NE levels (see Figures 3A-3C).

Because primary BAL macrophages and peripheral blood neutrophils do not culture well for extended periods, we then 
A

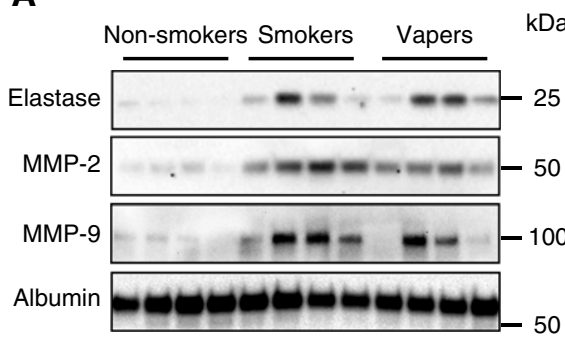

D

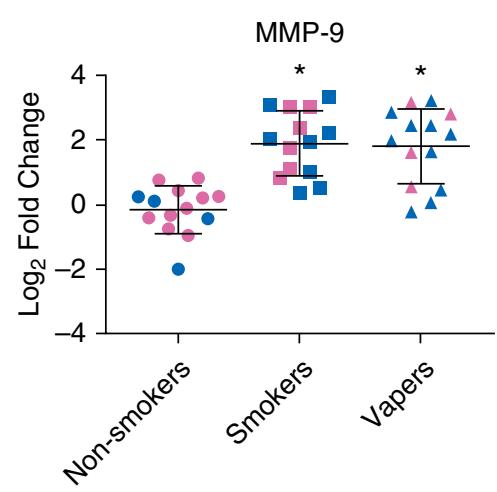

G

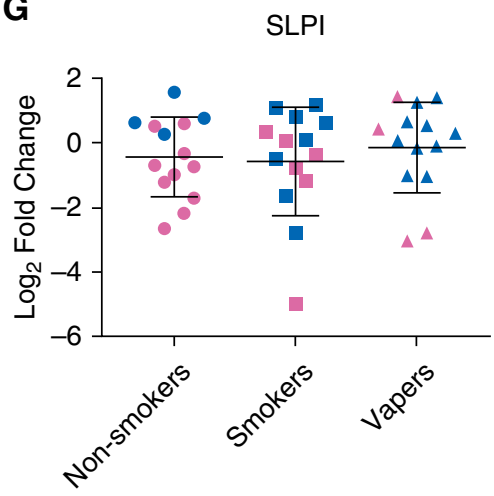

B

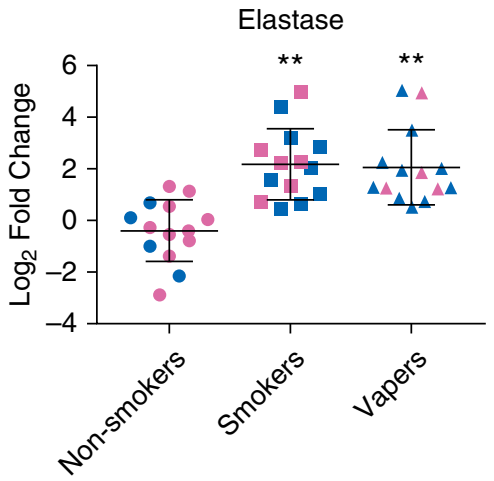

E

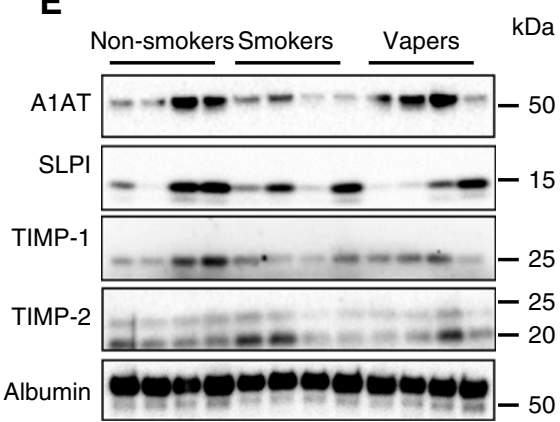

H

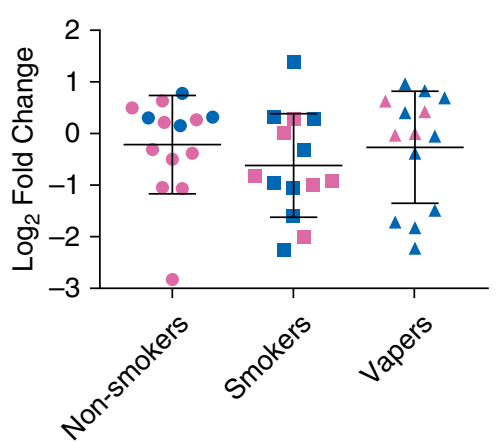

C
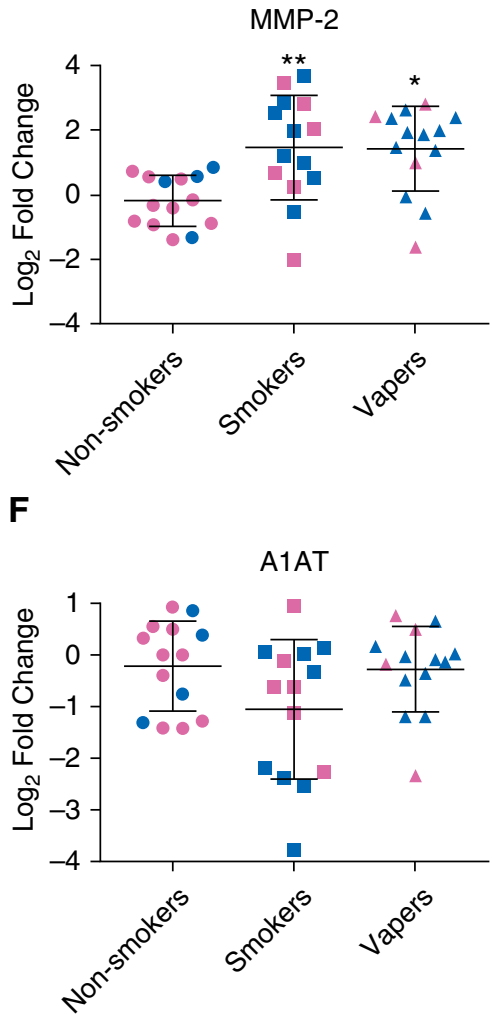

I

TIMP-2

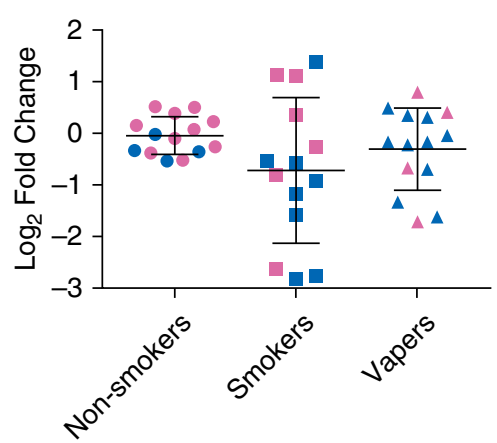

Figure 1. Protease protein levels are equally increased in vapers' and smokers' BAL. (A) Representative Western blots showing increased protease protein in smokers' and vapers' BAL, along with albumin as loading control. Full blots are provided in Figure E1. (B-D) Densitometric analysis of total protease levels normalized to albumin for neutrophil elastase (B), MMP-2 (matrix metalloprotease-2) $(C)$, and MMP-9 (D). (E) Representative Western blots of BAL protease inhibitors. (F-l) Densitometric analysis of BAL normalized to albumin control for A1AT (alpha-1 antitrypsin) ( $F$ ), SLPI (G), TIMP1 (tissue inhibitor of metalloproteinases-1) $(H)$, and TIMP2 ( $)$. Values are $\log _{2}$ fold change in smokers and vapers compared with age-matched nonsmokers; ${ }^{*} P \leqslant$ 0.05 and ${ }^{\star \star} P \leqslant 0.001$. Pink, female subjects; blue, male subjects. Data are shown as mean \pm SD. All $n=14$ subjects per group. Data were analyzed using ANOVA followed by Tukey's test. SLPI= secretory leukocyte protease inhibitor.

studied the effects of overnight incubation with $\mathrm{PG} / \mathrm{VG}$ plus or minus nicotine on differentiated Tohoku Hospital Pediatrics-1 cells, a macrophage-like cell line. We observed an increase in MMP-2/9 activity in the media after an overnight exposure of $1 \%$ PG/VG with $1.8 \%$ nicotine (Figure E4), suggesting that this phenomenon persisted after chronic exposure.

\section{Nicotine Increases Cytosolic $\mathbf{C a}^{2+}$ Levels and Induces Protease Release from BAL Macrophages and Peripheral Blood Neutrophils in a Dose-Dependent Manner}

Nicotinic acetylcholine receptors are cationpermeable, ligand-gated ion channels, and their stimulation by nicotine increases cytosolic $\mathrm{Ca}^{2+}$ levels (34). Importantly,
$\mathrm{Ca}^{2+}$ is a key second messenger that induces secretion of proteins, including proteases, into the extracellular environment. We exposed neutrophils and alveolar macrophages to a range of nicotine concentrations and determined the subsequent changes in cytoplasmic $\mathrm{Ca}^{2+}$ and protease secretion. Addition of nicotine to neutrophils rapidly increased 
A

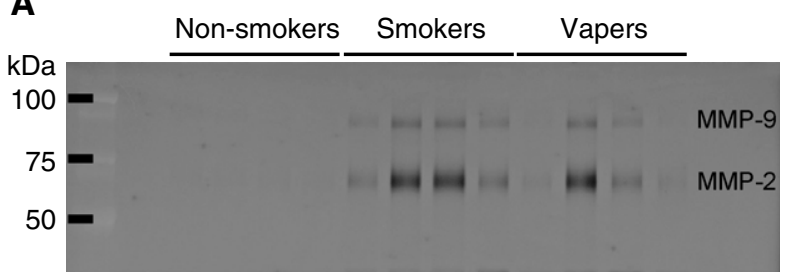

B Gelatinolytic Activity

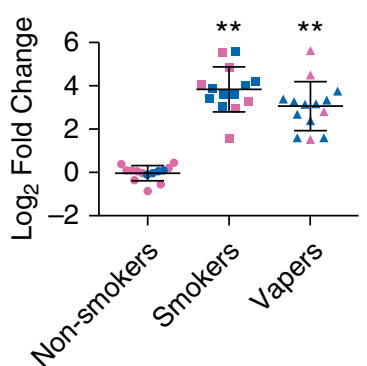

Figure 2. Total gelatinolytic activities are increased in vapers' BAL. Gelatinolytic activity was measured in BAL samples by zymography. $(A)$ Inverted image of representative zymogram showing increased MMP (matrix metalloprotease) activity in smokers' and vapers' BAL samples. (B) Quantification of gelatinolytic activity by MMP, shown as $\log _{2}$ fold change, compared with agematched nonsmokers. Individual activities of MMP-2 and MMP-9 are shown in Figure E4. ${ }^{*} P \leqslant$ 0.001 . Pink, female subjects; blue, male subjects. Data are shown as mean \pm SD. All $n=14$ subjects per group. Data were analyzed using ANOVA followed by Tukey's test.

cytoplasmic $\mathrm{Ca}^{2+}$ levels with a calculated $\mathrm{EC}_{50}$ of $210 \mu \mathrm{M}$ (Figures $4 \mathrm{~A}$ and $4 \mathrm{~B}$ ). Thapsigargin is a sarcoendoplasmic reticulum $\mathrm{Ca}^{2+}$-ATPase inhibitor that increases cytoplasmic $\mathrm{Ca}^{2+}$, which we used as a positive control (see Figure 4A). Thapsigargin increased cytoplasmic $\mathrm{Ca}^{2+}$ levels to similar extent as nicotine. We also evaluated NE release 3 hours after nicotine exposure. Nicotine stimulated NE release from neutrophils with an $\mathrm{EC}_{50}$ of $3.9 \mathrm{mM}$ (see Figure 4C). Macrophages showed more sensitivity to nicotine for both the $\mathrm{Ca}^{2+}$ response and protease release (see Figures 4D-4F). After nicotine addition, a rapid increase in cytosolic $\mathrm{Ca}^{2+}$ occurred with an $\mathrm{EC}_{50}$ of $96.4 \mu \mathrm{M}$ that was also similar in magnitude to the effects of thapsigargin (see Figures 4D and 4E). However, protease release from macrophages showed more sensitivity to nicotine, and the $\mathrm{EC}_{50}$ for MMP-2/9 release was $37.4 \mathrm{~nm}$ (see Figure 4F). Because both cell types showed dose-dependent responses to nicotine, we then used quantitative PCR to look for expression of several nicotinic acetylcholine receptors (nAChRs). Neutrophils expressed CHRNB1 (cholinergic receptors nicotinic subunit $\mathrm{B} 1$ ), CHRNA5, and CHRNA6 (see Figure 4G). In addition to these receptors, macrophages also expressed CHRNA7 (see Figure 4H).

\section{Evaluation of Nicotine, Cotinine, and Hydroxycotinine in the Lungs of Vapers}

Because nicotine was the key factor for protease release from inflammatory cells, we measured nicotine concentrations in vapers sputum under real-life conditions; that is, immediately after a vape session, using a mass spectrometry-based method (see online supplement). Table E6 shows the demographic details of the volunteers, their choice of e-liquid, and their vaping patterns before sputum induction. We focused on the identification of nicotine and its two major metabolites; namely, cotinine and hydroxycotinine (35). As expected, the amount of detectable nicotine, cotinine, and hydroxycotinine was negligible in the sputum from control subjects (Figures 5A-5C and see Table E6). In contrast, nicotine was significantly elevated in vapers sputum. Cotinine and hydroxycotinine were also significantly elevated but were present at lower levels, suggesting that significant nicotine metabolism did not occur in the lung lumen (see Figures 5A-5C). BAL samples from nonsmokers and vapers also exhibited significant amounts of nicotine and cotinine (see Figures E6A-E6C). Importantly, the mean nicotine concentration in sputum $(\sim 45 \mu \mathrm{M})$ (see Figure $5 \mathrm{~A})$ and BAL $(\sim 3 \mu \mathrm{M})$ (see Figure E6A) were sufficient to elicit protease release from macrophages (see Figure 4F).
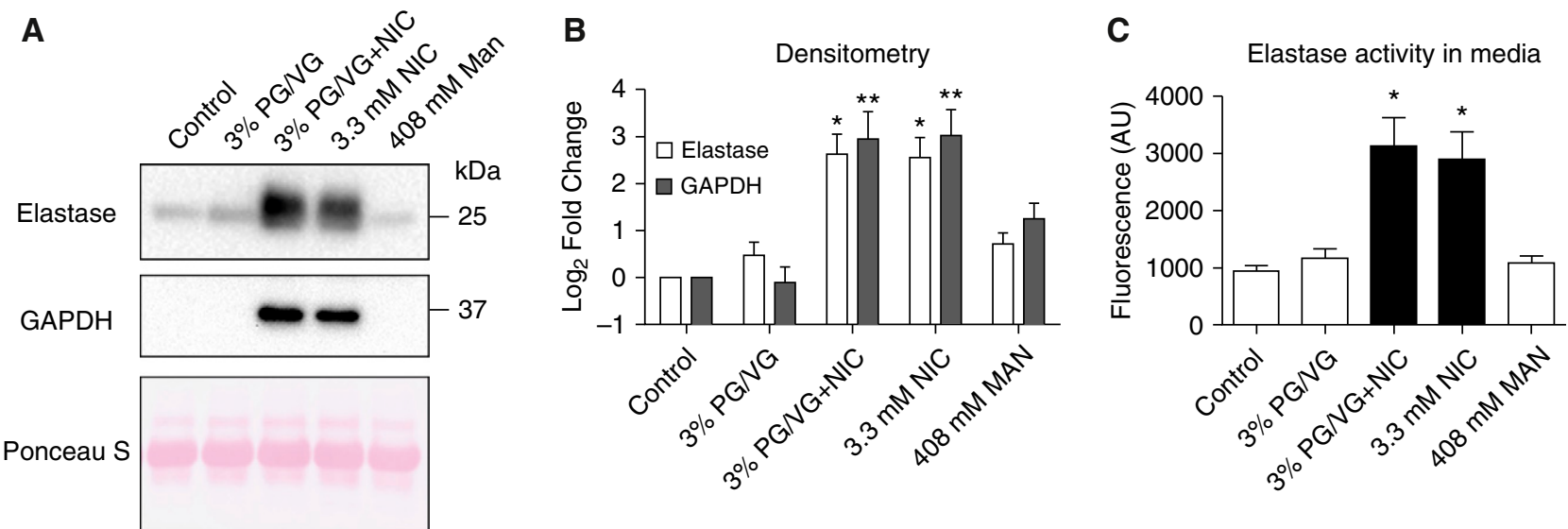

Figure 3. Nicotine, but not propylene glycol and vegetable glycerin (PG/VG), induces neutrophil elastase (NE) release from neutrophils. Neutrophils were isolated from peripheral blood and exposed to combinations of PG/VG, nicotine or mannitol (osmotic control) for 4 hours. (A) Representative Western blots of neutrophil secretions after incubation with the indicated e-liquid components, with Ponceau $S$ staining as a loading control. (B) Densitometric analysis of NE and GAPDH normalized to Ponceau S staining shown as fold change compared with control. (C) NE activity in concentrated media, as measured using the fluorogenic substrate assay. ${ }^{*} P \leqslant 0.05$ and ${ }^{* *} P \leqslant 0.001$. Data are shown as mean \pm SEM. All $n=12$ per group. Data were analyzed using ANOVA followed by Tukey's test. $\mathrm{AU}=$ arbitrary units; $\mathrm{MAN}=$ mannitol; $\mathrm{NIC}=$ nicotine. 
A Neutrophils

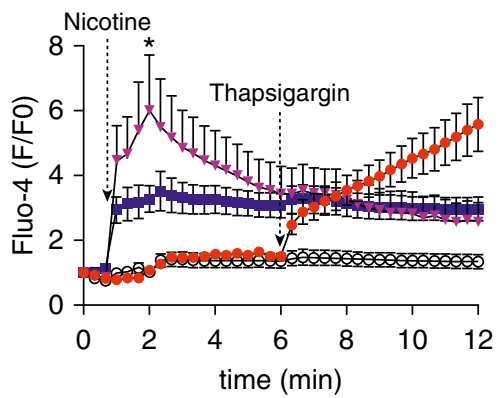

D

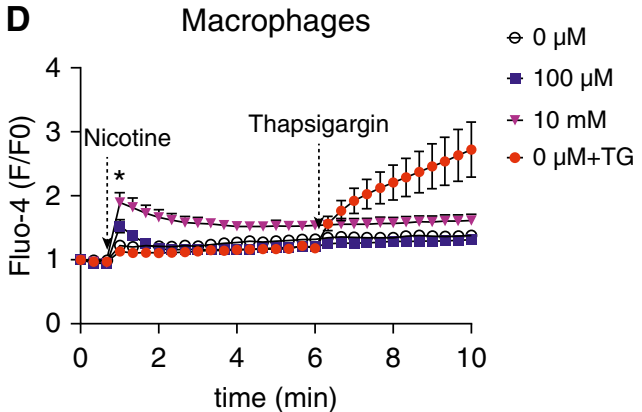

G

Neutrophils

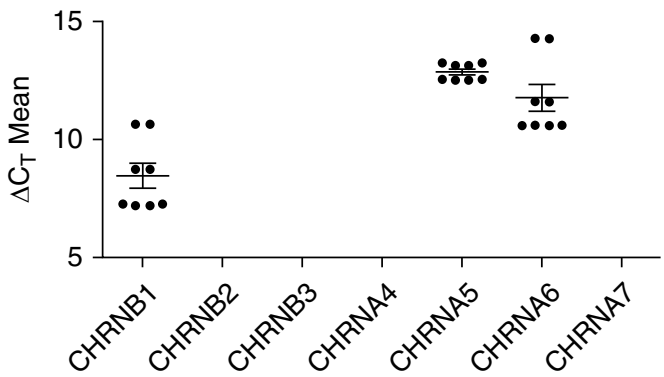

B

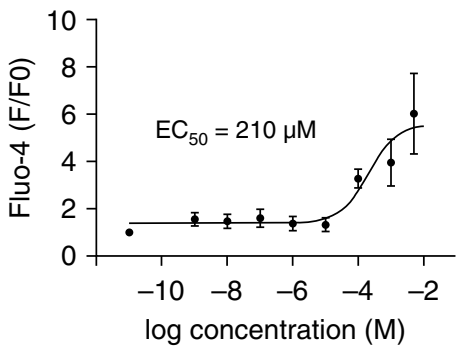

E

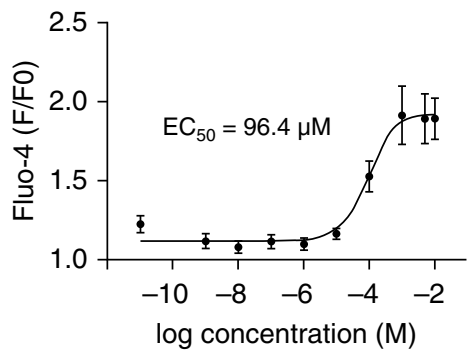

C

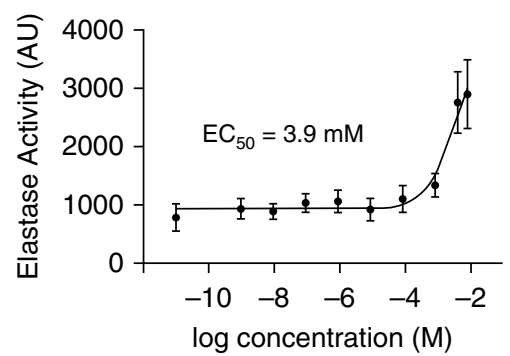

F

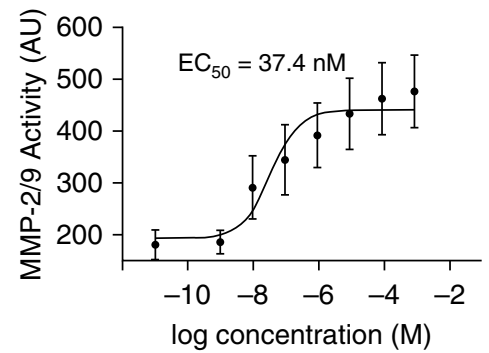

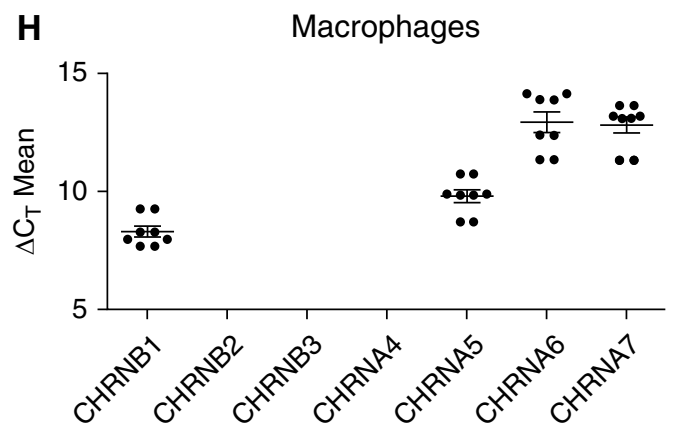

Figure 4. Nicotine causes dose-dependent increases in cytoplasmic $\mathrm{Ca}^{2+}$ and induces protease release from immune cells. Neutrophils and alveolar macrophages were loaded with the calcium indicator dye Fluo-4 direct and the change in fluorescence, as an indicator of cytoplasmic Ca ${ }^{2+}$, was recorded over time. The media was then sampled 4 hours after exposure to measure protease activity, as indicated by changes in cleavage of fluorogenic substrates specific to neutrophil elastase (NE) and MMP-2/9 (matrix metalloprotease-2/9). (A) Changes in Fluo4 fluorescence, as an indicator of cytoplasmic $\mathrm{Ca}^{2+}$ levels over time in peripheral blood neutrophils. $(B$ and $C$ ) Dose responses to nicotine exposure in neutrophils for peak (2 min) increases in cytoplasmic $\mathrm{Ca}^{2+}$ levels and 3 hours secreted NE activity, respectively; $n=9$ per group. Data were normalized to GAPDH expression; $n=8$ per group. (D) Changes in Fluo-4 fluorescence, as an indicator of cytoplasmic $\mathrm{Ca}^{2+}$ levels, in BAL macrophages. ( $E$ and $F$ ) Dose responses to nicotine exposure in BAL macrophages for peak $(2 \mathrm{~min})$ increases in cytoplasmic $\mathrm{Ca}^{2+}$ levels after nicotine exposure and secreted NE activity, respectively; $n=9$ per group. (G) Nicotinic acetylcholine receptor expression in peripheral blood neutrophils, as measured by quantitative PCR (qPCR). (H) Nicotinic acetylcholine receptor expression in alveolar macrophages, as measured by qPCR; $n=8$ per group. ${ }^{*} P \leqslant 0.05$ compared with control. Data are shown as mean \pm SEM. Data were analyzed using Mann-Whitney $U$ test $(A$ and $E)$ or fit to the equation $Y=$ bottom $+($ top - bottom $) /\left(1+10^{X-\log I C 50}\right)(B, C, F$, and $G)$. $A U=$ arbitrary unit; $\Delta \mathrm{C}_{\mathrm{T}}=$ delta cycle threshold; $\mathrm{CHRN}=$ cholinergic receptors nicotinic subunit; $E C=$ effective concentration; $F / F O=$ ratio of final to initial fluorescence; $T G=$ thapsigargin .

\section{Discussion}

Here, we have focused on the effects of vaping e-cigarettes on pulmonary

(i.e., BAL) protease levels because the link between increased proteolysis and disease is well established (36). NE is a serine protease with a broad substrate specificity that is important for host defense. We found that both total NE protein levels and NE functional activity were elevated in both smokers and vapers (see Figures $1 \mathrm{~A}$ and $1 \mathrm{~B}$ and $\mathrm{E} 3$ ). In contrast, the NE secretory leukocyte protease inhibitor was unchanged, indicating that the increased NE-dependent proteolysis was due to higher NE levels rather than a decrease in inhibitor levels. Importantly, the elevation in NE levels was 1) similar between vapers and smokers (see Figures
$1 \mathrm{~A}$ and $1 \mathrm{~B}$ ) and 2) was also seen in vapers who were never-smokers (see Figure E2), indicating that this change was caused by chronic vaping and was not simply a residual effect from smoking. Elevated lung NE levels and activity are seen in alpha-1 antitrypsin deficiency, an inherited condition in which individuals lack a key protease inhibitor (i.e., alpha-1 antitrypsin). 
A

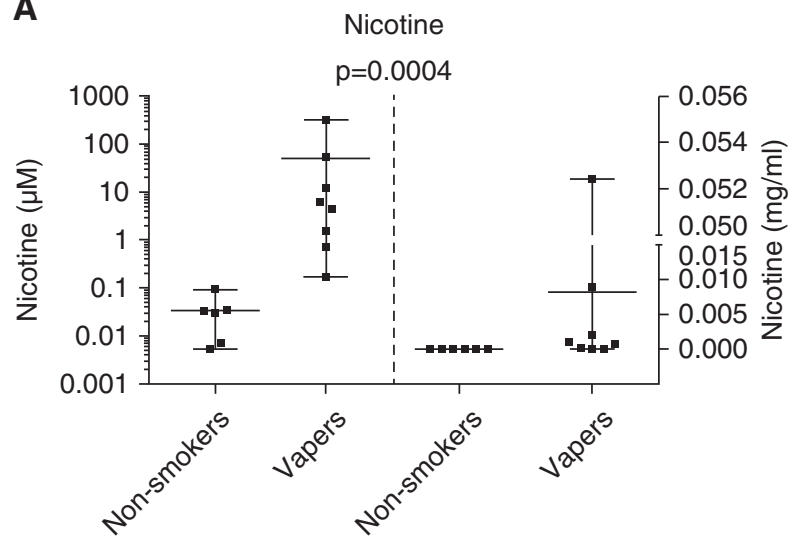

B

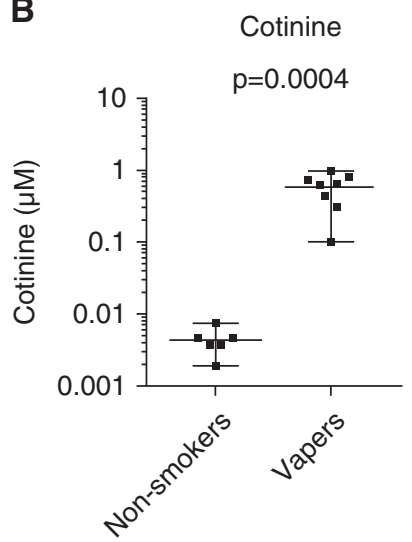

C

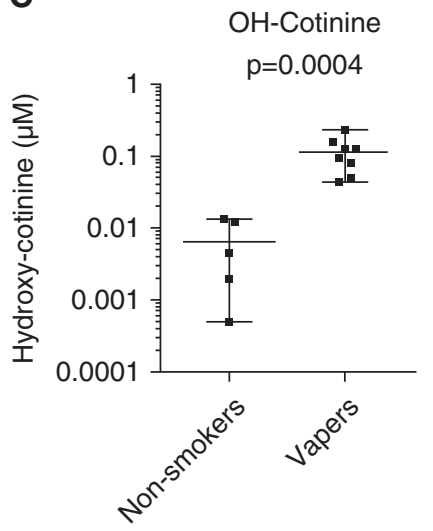

Figure 5. Measurements of nicotine and its metabolites in vapers' sputum. Vapers were asked to vape normally over 1 hour, and sputum was induced immediately afterwards. Metabolite levels were then measured by gas chromatography-mass spectrometry. ( $A-C$ ) Graphs show concentrations of nicotine $(A)$, cotinine $(B)$, and hydroxycotinine $(C)$, from control subjects $(n=6)$ and vapers $(n=8)$. Nicotine concentrations are shown as both micromolar (left) and milligrams per milliliter (right). Data are shown as mean with range along with individual data points and $P$ values. Data were analyzed using the Mann-Whitney $U$ test. $\mathrm{OH}$-Cotinine = hydroxycotinine.

Patients with alpha-1 antitrypsin deficiency typically develop COPD-like symptoms, including emphysema, by their mid-40s (26). Due to unremittent infection and inflammation, NE levels are also extremely high in the lungs of patients with cystic fibrosis, and this increase correlates with a greater decline in $\mathrm{FEV}_{1}$ (37). Furthermore, instillation of supernatant from cystic fibrosis sputum into rat lungs caused rapid hemorrhaging that was attenuated by pretreatment with a NE inhibitor, thus illustrating the damaging effect of high NE levels (38). NE levels are also upregulated in patients with COPD (39). Interestingly, NE knockout $\left(\mathrm{ELANE}^{-1-}\right.$ ) mice were protected from developing emphysema despite being exposed to chronic tobacco smoke, which also indicates a causal link between NE and lung damage (18).

We also detected increased MMP2 and MMP-9 levels and activity in vapers' BAL using 3 independent techniques; namely, Western blotting, the fluorogenic substrate assay, and by zymography (see Figures 1A-1D, 2A-2C, and E3). Again, despite being elevated, MMP-2 and MMP-9 levels were not different between smokers and vapers, and were seen at similar levels in vapers who never smoked (see Figures 1A-1D, 2A-2C, and E2). MMP-2 and MMP-9 (also known as gelatinase $\mathrm{a}$ and $\mathrm{b}$, respectively) are also implicated in disease. MMP-2 levels are elevated in smokers, especially in those with COPD, and this increase inversely correlates with $\mathrm{FEV}_{1}(40,41)$. Wells and colleagues demonstrated elevated MMP-9 levels in sputum from patients with COPD that correlated with an increased incidence of acute exacerbation (42). MMP-2 and MMP-9 levels are also upregulated in sputum from people with asthma (43) and MMP-9 is elevated in BAL from people with cystic fibrosis, which also inversely correlates with lung function (44). Interestingly, serum MMP-9 levels also correlate with COPD disease severity (45). Thus, protease levels, and MMPs in particular, may be a useful biomarker for chronic vaping that can be applied to larger populations.

To date, the effects of chronic e-cigarette use on the lung are poorly characterized. Reidel and colleagues recently performed proteomics on vapers' sputum (31). Sputum reflects a different compartment than BAL and is more representative of the surfaces of central and large airways, rather than the small distal airways and alveolar regions. However, these researchers found more protein changes in vapers' than in smokers' sputum relative to nonsmokers. Importantly, they detected upregulation of NE and MMP-9 protein levels in vapers' sputum, although they did not measure protease activity. Their data also suggested that vapers' neutrophils were altered, and they found increased evidence of neutrophil extracellular trap release, socalled NETosis, in vapers' neutrophils. In this previous study, all of the vapers were ex-smokers and there is the possibility that some effects seen in vapers were due to the previous tobacco exposure. However, Garcia-Arcos and colleagues exposed naïve mice to $P G / V G$ and nicotine for 4 months (46), which caused significant emphysema. Although they did not measure murine lung protease activity per se, tobacco exposure-induced emphysema is dependent on increased proteolysis (46), making it likely that vaping increased protease activity in these mice.

To better understand how vaping led to increased proteolysis, we exposed inflammatory cells to various e-liquid components in vitro. Nicotine induced protease release from neutrophils that was independent of PG/VG and was not an osmotic effect (see Figure 3). Interestingly, we detected GAPDH in the culture media, suggesting that some cell lysis may have occurred. Although the interaction between nicotine and neutrophils has not been extensively studied, nicotine has been previously shown to increase NE gene and protein expression in human leukemic-60 cells (47, 48). Garcia-Arcos and colleagues concluded that vape-induced emphysema was driven by nicotine rather than by other e-liquid components such as PG/VG (46). Similarly, Scott and colleagues found that nicotine contained in e-cigarettes exerted proinflammatory effects on human alveolar macrophages (49). In vitro exposure of neutrophils to nicotine has also been shown to increase NE gene expression and azurophilic degranulation $(47,50)$. Nicotine 
has also been shown to cause upregulation of MMP-2 and MMP-9 in the murine macrophage cell line RAW 264.7 (51). Thus, it is likely that nicotine is directly exerting effects on multiple immune cell types.

$\mathrm{Ca}^{2+}$ is an important second messenger that, when elevated, can acutely trigger secretion of proteins, including proteases and cytokines $(52,53)$.

Interaction between nicotine and $\mathrm{nAChR}$ leads to cellular $\mathrm{Ca}^{2+}$ influx (54). Consistent with this well-established interaction between nicotine and its receptor, here we found that nicotine elicited an increase in cytoplasmic $\mathrm{Ca}^{2+}$ in both neutrophils and macrophages with $\mathrm{EC}_{50} \mathrm{~s}$ of 210 and $94 \mu \mathrm{M}$, respectively. We also demonstrated that nicotine caused an increase in protease activity in the media of both cell types. Interestingly, the $\mathrm{EC}_{50}$ for protease release from neutrophils $(3.9 \mathrm{mM})$ was somewhat higher than for the $\mathrm{Ca}^{2+}$ response $(210 \mu \mathrm{M})$ and, given that we observed GAPDH in the media, we speculate that protease release from neutrophils may have occurred subsequent to cell lysis and NETosis. However, the $\mathrm{EC}_{50}$ for protease release from alveolar macrophages was approximately $40 \mathrm{~nm}$, suggesting that we had observed regulated, $\mathrm{Ca}^{2+}$-dependent protease secretion, rather than lysis (see Figure 4). Although both cell types expressed several subtypes of nAChR, only alveolar macrophages expressed CHRNA7 (see Figure 4). Although many nAChRs express as heteromultimers, CHRNA7 expresses as a homotetramer, and we propose that its presence leads to the increased sensitivity to nicotine in macrophages. Of note, CHRNA7 has recently been shown to modulate the lung's response to inflammation and tobacco smoke in a murine model, suggesting that its expression is physiologically relevant during the inflammatory response to tobacco smoke (55).

The nicotine concentration in serum and urine has been well studied (56). However, considerably less is known about nicotine levels in the lung immediately after smoking or vaping. We previously found that nicotine in cigarette smokers' sputum was approximately $30 \mu \mathrm{M}$ within approximately 30 minutes after smoking (57). Because the levels of vape and nicotine exposure in vapers' lungs were unknown, we measured nicotine within a similar timeframe (i.e., $\sim 30 \mathrm{~min}$ ) after vaping. Here, we studied both sputum and BAL. We found that vaping led to a significant increase in nicotine and other metabolites, and that nicotine was present at a similar level as seen in smokers' sputum $(\sim 50 \mu \mathrm{M})$. Nicotine was also present in vapers' BAL at approximately $3 \mu \mathrm{M}$. Nicotine is extremely concentrated in e-liquids and, in our study, freebase nicotine ranged from 3 to $12 \mathrm{mg} / \mathrm{ml}$, which equates to 18.52 to $74.07 \mathrm{mM}$. Uncharged nicotine is cell-permeable, which is how it reaches systemic circulation. Thus, it is likely that we are measuring nicotine as it is being transepithelially absorbed down its chemical gradient into the body, and we speculate that much higher levels of nicotine are initially seen in vapers' airways during actual inhalation; that is, in the period before we could induce sputum. Furthermore, sputum and BAL were obtained at least 30 minutes and 2 hours, respectively, after the subjects' last vape sessions, as necessitated by the sputum induction and bronchoscopy procedures, which may explain the lower nicotine levels. Importantly, the concentration of nicotine measured in vapers' airways was sufficient to elicit protease release from relevant immune cell types in comparison (see Figures 4, 5, and E6). Our data implicate nicotine in the release of potentially damaging proteases into the lung lumen. Beyond the potential for altering the lung, nicotine is also highly addictive and desensitization of $\mathrm{nAChR}$ in the brain provides nicotine's psychotropic effects Of concern is the increase in vaping in adolescents, suggesting that more young people are being exposed to nicotine (58). Crucially, nicotine affects neuronal development in adolescents (59) and can also serve as a gateway to further addition. For example, in a murine model, pretreatment with nicotine increased the propensity for cocaine addiction but not vice versa, suggesting that this is a nicotinespecific effect (60).

There are a few limitations of our study. First, although we were adequately powered, the number of subjects in this study was relatively small and, owing to the potential importance of our findings, we propose that lung protease levels need to be assessed in a larger population.
Second, for the in vitro portion of the study, we studied only two cell types (see Figures 3, 4, and E5) and other cell types, including airway epithelia, may also contribute to nicotine-induced protease release $(61,62)$. Finally, some vapers in our study were ex-smokers and, although they switched from combustible tobacco to e-cigarettes at least 6 months prior to sample collection, there may be a residual effect of smoking. As a counterpoint to this, Zhang and colleagues previously demonstrated that many genes return to baseline after smoking cessation (63), suggesting that the study of former smokers is valid. Despite these potential limitations, increased protease levels and activities were observed in e-cigarette users regardless of their former smoking status, suggesting that continued vaping may be a significant risk to pulmonary health.

Indeed, given the role of proteases in lung damage and remodeling, it is possible that chronic vaping may pose a risk of emphysema and/or bronchiectasis. Because these ultrastructural changes often occur before changes can be detected by pulmonary function tests, we also propose that healthy, chronic vapers be studied using lung-imaging techniques to better understand the potential risk that vaping may pose to lung health. Given the established link between proteolysis and lung disease, long-term studies on the effects of continued use of e-cigarettes should be conducted before prescribing them as a safer alternative to smoking. Certainly, from a lung perspective, and based on lung proteases as an endpoint, it is hard to understand how they can be classified as being 95\% safer than tobacco smoking, as has previously been stated (64).

Author disclosures are available with the text of this article at www.atsjournals.org.

Acknowledgment: The authors thank Eric S Davis, Martha A. Almond, Carol A. Robinette, Heather B. Wells, Joleen Soukup, Tara N. G. Lee, and the UNC CF Center Molecular Core for technical assistance. They also thank the UNC Center for Global Initiatives for their support. This work was funded by $\mathrm{NIH} / \mathrm{FDA} \mathrm{HL} 120100$ and $\mathrm{NIH} / \mathrm{NHLBI} \mathrm{HL} 135642$. Research reported in this publication was in part supported by $\mathrm{NIH}$ and the US Food and Drug Administration Center for Tobacco Products. The content is solely the responsibility of the authors and does not necessarily represent the official views of the $\mathrm{NIH}$ or the US Food and Drug Administration. 


\section{References}

1. WHO. World health statistics 2018: monitoring health for the SDGs, sustainable development goals. Geneva, Switzerland: World Health Organization; 2018.

2. Prochaska JJ, Benowitz NL. The past, present, and future of nicotine addiction therapy. Annu Rev Med 2016;67:467-486.

3. Siegel MB, Tanwar KL, Wood KS. Electronic cigarettes as a smokingcessation: tool results from an online survey. Am J Prev Med 2011;40: 472-475.

4. Kalkhoran S, Glantz SA. E-cigarettes and smoking cessation in realworld and clinical settings: a systematic review and meta-analysis. Lancet Respir Med 2016;4:116-128.

5. Bullen C, Howe C, Laugesen M, McRobbie H, Parag V, Williman J, et al. Electronic cigarettes for smoking cessation: a randomised controlled trial. Lancet 2013;382:1629-1637.

6. Newton JN, Dockrell M, Marczylo T. Making sense of the latest evidence on electronic cigarettes. Lancet 2018;391: 639-642.

7. East K, Brose LS, McNeill A, Cheeseman H, Arnott D, Hitchman SC. Harm perceptions of electronic cigarettes and nicotine: a nationally representative cross-sectional survey of young people in Great Britain. Drug Alcohol Depend 2018;192:257-263.

8. Jankowski M, Brożek G, Lawson J, Skoczyński S, Zejda JE. E-smoking: emerging public health problem? Int J Occup Med Environ Health 2017;30:329-344.

9. Hartmann-Boyce J, McRobbie H, Bullen C, Begh R, Stead LF, Hajek P. Electronic cigarettes for smoking cessation. Cochrane Database Syst Rev 2016;9:CD010216.

10. Bals R, Boyd J, Esposito S, Foronjy R, Hiemstra PS, Jimenez-Ruiz CA, et al. Electronic cigarettes: a task force report from the European Respiratory Society. Eur Respir J 2019;53:1801151.

11. McNeill A, Brose LS, Calder R, Bauld L, Robson D. Evidence review of e-cigarettes and heated tobacco products 2018: a report commissioned by Public Health England. London, United Kingdom: Public Health England; 2018.

12. Rogers LD, Overall CM. Proteolytic post-translational modification of proteins: proteomic tools and methodology. Mol Cell Proteomics 2013;12:3532-3542.

13. Chondrogianni N, Petropoulos I, Grimm S, Georgila K, Catalgol B, Friguet $\mathrm{B}$, et al. Protein damage, repair and proteolysis. Mol Aspects Med 2014;35:1-71.

14. Abboud RT, Vimalanathan S. Pathogenesis of COPD: part I. The role of protease-antiprotease imbalance in emphysema. Int $J$ Tuberc Lung Dis 2008;12:361-367.

15. Skrzydlewska E, Sulkowska M, Koda M, Sulkowski S. Proteolyticantiproteolytic balance and its regulation in carcinogenesis. World $\mathrm{J}$ Gastroenterol 2005;11:1251-1266.

16. Nadel JA. Role of neutrophil elastase in hypersecretion during COPD exacerbations, and proposed therapies. Chest 2000;117(Suppl 2): 386S-389S

17. Fischer BM, Pavlisko E, Voynow JA. Pathogenic triad in COPD: oxidative stress, protease-antiprotease imbalance, and inflammation. Int J Chron Obstruct Pulmon Dis 2011;6:413-421.

18. Shapiro SD, Goldstein NM, Houghton AM, Kobayashi DK, Kelley D, Belaaouaj A. Neutrophil elastase contributes to cigarette smoke-induced emphysema in mice. Am J Pathol 2003;163: 2329-2335.

19. Churg A, Cosio M, Wright JL. Mechanisms of cigarette smoke-induced COPD: insights from animal models. Am J Physiol Lung Cell Mol Physiol 2008;294:L612-L631.

20. Antunes MA, Rocco PR. Elastase-induced pulmonary emphysema: insights from experimental models. An Acad Bras Cienc 2011;83: 1385-1396.

21. Muley T, Wiebel M, Schulz V, Ebert W. Elastinolytic activity of alveolar macrophages in smoking-associated pulmonary emphysema. Clin Investig 1994;72:269-276.

22. Harris JO, Olsen GN, Castle JR, Maloney AS. Comparison of proteolytic enzyme activity in pulmonary alveolar macrophages and blood leukocytes in smokers and nonsmokers. Am Rev Respir Dis 1975;111:579-586.
23. Takahashi H, Ishidoh K, Muno D, Ohwada A, Nukiwa T, Kominami E, et al. Cathepsin L activity is increased in alveolar macrophages and bronchoalveolar lavage fluid of smokers. Am Rev Respir Dis 1993; 147:1562-1568

24. Weitz JI, Crowley KA, Landman SL, Lipman BI, Yu J. Increased neutrophil elastase activity in cigarette smokers. Ann Intern Med 1987:107:680-682.

25. Sohal SS, Reid D, Soltani A, Ward C, Weston S, Muller HK, et al. Reticular basement membrane fragmentation and potential epithelia mesenchymal transition is exaggerated in the airways of smokers with chronic obstructive pulmonary disease. Respirology 2010;15: 930-938.

26. Crystal RG. Alpha 1-antitrypsin deficiency, emphysema, and liver disease: genetic basis and strategies for therapy. J Clin Invest 1990; 85:1343-1352.

27. Stick SM, Brennan S, Murray C, Douglas T, von Ungern-Sternberg BS, Garratt LW, et al.; Australian Respiratory Early Surveillance Team for Cystic Fibrosis (AREST CF). Bronchiectasis in infants and preschool children diagnosed with cystic fibrosis after newborn screening. $J$ Pediatr 2009;155:623-628, e1.

28. Mason SD, Joyce JA. Proteolytic networks in cancer. Trends Cell Biol 2011;21:228-237.

29. Ghosh A, Coakley RC, Mascenik T, Rowell TR, Davis ES, Rogers K et al. Chronic E-cigarette exposure alters the human bronchial epithelial proteome. Am J Respir Crit Care Med 2018;198:67-76.

30. Martin EM, Clapp PW, Rebuli ME, Pawlak EA, Glista-Baker E, Benowitz $\mathrm{NL}$, et al. E-cigarette use results in suppression of immune and inflammatory-response genes in nasal epithelial cells similar to cigarette smoke. Am J Physiol Lung Cell Mol Physiol 2016;311: L135-L144.

31. Reidel B, Radicioni G, Clapp PW, Ford AA, Abdelwahab S, Rebuli ME, et al. E-cigarette use causes a unique innate immune response in the lung, involving increased neutrophilic activation and altered mucin secretion. Am J Respir Crit Care Med 2018;197:492-501.

32. Zhang Y, Sumner W, Chen DR. In vitro particle size distributions in electronic and conventional cigarette aerosols suggest comparable deposition patterns. Nicotine Tob Res 2013:15:501-508.

33. Sibille Y, Reynolds HY. Macrophages and polymorphonuclear neutrophils in lung defense and injury. Am Rev Respir Dis 1990;141: 471-501.

34. Zia S, Ndoye A, Nguyen VT, Grando SA. Nicotine enhances expression of the alpha 3 , alpha 4 , alpha 5 , and alpha 7 nicotinic receptors modulating calcium metabolism and regulating adhesion and motility of respiratory epithelial cells. Res Commun Mol Pathol Pharmacol 1997;97:243-262.

35. Benowitz NL, Hukkanen J, Jacob P III. Nicotine chemistry, metabolism, kinetics and biomarkers. Handb Exp Pharmacol 2009;(192):29-60.

36. Ferkol T, Schraufnagel D. The global burden of respiratory disease. Ann Am Thorac Soc 2014;11:404-406.

37. Sagel SD, Sontag MK, Wagener JS, Kapsner RK, Osberg I, Accurso FJ. Induced sputum inflammatory measures correlate with lung function in children with cystic fibrosis. J Pediatr 2002;141:811-817.

38. Rees DD, Brain JD. Effects of cystic fibrosis airway secretions on rat lung: role of neutrophil elastase. Am J Physiol 1995;269: L195-L202.

39. Stockley RA. Neutrophils and the pathogenesis of COPD. Chest 2002; 121(Suppl):151S-155S.

40. Baraldo S, Bazzan E, Zanin ME, Turato G, Garbisa S, Maestrelli P, et al Matrix metalloproteinase-2 protein in lung periphery is related to COPD progression. Chest 2007;132:1733-1740.

41. Chen Y, Chen P, Hanaoka M, Droma Y, Kubo K. Enhanced levels of prostaglandin E2 and matrix metalloproteinase-2 correlate with the severity of airflow limitation in stable COPD. Respirology 2008;13: 1014-1021.

42. Wells JM, Parker MM, Oster RA, Bowler RP, Dransfield MT, Bhatt SP, et al. Elevated circulating MMP-9 is linked to increased COPD exacerbation risk in SPIROMICS and COPDGene. JCI Insight 2018;3: e123614.

43. Cataldo D, Munaut C, Noël A, Frankenne F, Bartsch P, Foidart JM, et al MMP-2- and MMP-9-linked gelatinolytic activity in the sputum from patients with asthma and chronic obstructive pulmonary disease. Int Arch Allergy Immunol 2000;123:259-267. 
44. Sagel SD, Kapsner RK, Osberg I. Induced sputum matrix metalloproteinase- 9 correlates with lung function and airway inflammation in children with cystic fibrosis. Pediatr Pulmonol 2005; 39:224-232.

45. Brajer B, Batura-Gabryel H, Nowicka A, Kuznar-Kaminska B, Szczepanik A. Concentration of matrix metalloproteinase-9 in serum of patients with chronic obstructive pulmonary disease and a degree of airway obstruction and disease progression. J Physiol Pharmacol 2008;59:145-152.

46. Garcia-Arcos I, Geraghty P, Baumlin N, Campos M, Dabo AJ, Jundi B, et al. Chronic electronic cigarette exposure in mice induces features of COPD in a nicotine-dependent manner. Thorax 2016;71:1119-1129.

47. Armstrong LW, Rom WN, Martiniuk FT, Hart D, Jagirdar J, Galdston M. Nicotine enhances expression of the neutrophil elastase gene and protein in a human myeloblast/promyelocyte cell line. Am J Respir Crit Care Med 1996;154:1520-1524.

48. Wang XL, Kita K, Takahashi S, Hasegawa R, Chi XJ, Yamamori H, et al. Protease activity induced by nicotine in human cells. Int $\mathrm{J} \mathrm{Mol} \mathrm{Med}$ 1999;4:537-540.

49. Scott A, Lugg ST, Aldridge K, Lewis KE, Bowden A, Mahida RY, et al. Pro-inflammatory effects of e-cigarette vapour condensate on human alveolar macrophages. Thorax 2018;73:1161-1169.

50. Seow WK, Thong YH, Nelson RD, MacFarlane GD, Herzberg MC. Nicotine-induced release of elastase and eicosanoids by human neutrophils. Inflammation 1994;18:119-127.

51. Li ZZ, Guo ZZ, Zhang Z, Cao QA, Zhu YJ, Yao HL, et al. Nicotineinduced upregulation of VCAM-1, MMP-2, and MMP-9 through the $\alpha 7-n A C h R-J N K$ pathway in RAW264.7 and MOVAS cells. $\mathrm{Mol}$ Cell Biochem 2015;399:49-58.

52. Krüger B, Albrecht E, Lerch MM. The role of intracellular calcium signaling in premature protease activation and the onset of pancreatitis. Am J Pathol 2000;157:43-50.

53. Brown DM, Donaldson K, Borm PJ, Schins RP, Dehnhardt M, Gilmour $\mathrm{P}$, et al. Calcium and ROS-mediated activation of transcription factors and TNF-alpha cytokine gene expression in macrophages exposed to ultrafine particles. Am J Physiol Lung Cell Mol Physiol 2004;286:L344-L353.

54. Carlisle DL, Hopkins TM, Gaither-Davis A, Silhanek MJ, Luketich JD, Christie NA, et al. Nicotine signals through muscle-type and neurona nicotinic acetylcholine receptors in both human bronchial epithelial cells and airway fibroblasts. Respir Res 2004;5:27.
55. Gahring LC, Myers EJ, Dunn DM, Weiss RB, Rogers SW. Lung eosinophilia induced by house dust mites or ovalbumin is modulated by nicotinic receptor $\alpha 7$ and inhibited by cigarette smoke. Am J Physiol Lung Cell Mol Physiol 2018;315:L553-L562.

56. Jacob P III, Yu L, Duan M, Ramos L, Yturralde O, Benowitz NL. Determination of the nicotine metabolites cotinine and trans-3' hydroxycotinine in biologic fluids of smokers and non-smokers using liquid chromatography-tandem mass spectrometry: biomarkers for tobacco smoke exposure and for phenotyping cytochrome P450 2A6 activity. J Chromatogr B Analyt Technol Biomed Life Sci 2011; 879:267-276.

57. Clunes LA, Bridges A, Alexis N, Tarran R. In vivo versus in vitro airway surface liquid nicotine levels following cigarette smoke exposure. $J$ Anal Toxicol 2008;32:201-207.

58. Cullen KA, Ambrose BK, Gentzke AS, Apelberg BJ, Jamal A, King BA. Notes from the field: use of electronic cigarettes and any tobacco product among middle and high school students. United States, 2011-2018. MMWR Morb Mortal Wkly Rep 2018;67: 1276-1277.

59. Goriounova NA, Mansvelder HD. Short- and long-term consequences of nicotine exposure during adolescence for prefrontal cortex neuronal network function. Cold Spring Harb Perspect Med 2012;2 a012120.

60. Levine A, Huang Y, Drisaldi B, Griffin EA Jr, Pollak DD, Xu S, et al. Molecular mechanism for a gateway drug: epigenetic changes initiated by nicotine prime gene expression by cocaine. Sci Trans/ Med 2011;3:107ra109.

61. Oei E, Kalb T, Beuria P, Allez M, Nakazawa A, Azuma M, et al. Accessory cell function of airway epithelial cells. Am J Physiol Lung Cell Mol Physiol 2004;287:L318-L331.

62. Faiz A, Tjin G, Harkness L, Weckmann M, Bao S, Black JL, et al. The expression and activity of cathepsins $\mathrm{D}, \mathrm{H}$ and $\mathrm{K}$ in asthmatic airways. PLoS One 2013;8:e57245.

63. Zhang L, Lee JJ, Tang H, Fan YH, Xiao L, Ren H, et al. Impact of smoking cessation on global gene expression in the bronchial epithelium of chronic smokers. Cancer Prev Res (Phila) 2008;1: 112-118.

64. Nutt DJ, Phillips LD, Balfour D, Curran HV, Dockrell M, Foulds J, et al. Estimating the harms of nicotine-containing products using the MCDA approach. Eur Addict Res 2014;20: 218-225. 